\title{
Génie génétique, production de médicaments et thérapie génique
}

\begin{abstract}
Production par génie génétique de protéines d'intérêt biologique et thérapeutique.

La purification en grande quantité d'hormones peptidiques, de facteurs de coagulation, de protéines vaccinales... ou de tout autre polypeptide d'intérêt biologique (interférons, interleukines, facteurs de croissance, etc.), est le domaine privilégié des biotechnologies utilisant les méthodes des recombinants d'ADN, ou "génie génétique ". Le principe de ces méthodes est de transférer un gène naturel "cloné " et plus ou moins modifié in vitro, voire même un gène artificiel synthétisé chimiquement, dans une cellule vivante qui servira à l'" exprimer " en la protéine biologiquement active désirée.
\end{abstract}

Les trois exigences requises pour entreprendre une telle étude sont celles de l'activité du produit obtenu, de son innocuité et de sa production avec un rendement et à des coûts en faisant une méthode concurrentielle de celles, plus classiques, consistant à l'extraire de tissus humains ou animaux.

L'aspect quantitatif du problème exige la construction de gènes hybrides auxquels ont été rajoutées différentes séquences exogènes destinées à augmenter la transcription du gène, la stabilité du messager (et parfois de la protéine), sa traductibilité et, chaque fois que possible, à permettre l'exportation de la protéine dans le milieu extra-cellulaire (figure 1, page suivante). L'innocuité du produit requiert, notamment s'il s'agit d'un facteur de substitution à administrer à des malades, que soient éliminées de la protéine à utiliser toutes séquences protéiques potentiellement immunogènes. L'activité biologique exige enfin dans un certain nombre de cas que la protéine synthétisée subisse toute une série de modifications post-traductionnelles transformant un précurseur inactif en principe biologiquement actif : glycosylation, carboxylation, coupure protéolytique, par exemple. Or, seules les cellules eucaryotiques, et parfois même des cellules animales différenciées, sont capables d'effectuer ces opérations. En l'absence de modifications postsynthétiques indispensables, les bactéries (et surtout $E$. coli) sont les meilleures cellules productrices, peut-être concurrencées par les levures qui sont elles, en plus, capables d'effectuer certaines opérations de maturation posttraductionnelle (comme certains types de glycosylation).

Les cellules animales sont plus difficiles à cultiver en très grande quantité et produisent moins de la protéine désirée. Elles sont cependant le seul système utilisable dans des cas comme la production de facteurs de coagulation.

Thérapie génique: tendances pour demain.

Quoique la "chirurgie " et la " greffe " des gènes n'aient encore jamais été réalisées chez l'homme, il nous a semblé difficile de clore un " lexique " consacré à la génétique moléculaire et à la médecine sans au moins évoquer cet aspect.
La manipulation génétique d'embryons dans le but de remplacer un gène absent ou défectueux est techniquement concevable (quoique probablement très difficile) mais se heurte à de redoutables problèmes éthiques. Plus que le transfert direct de gènes dans l'œuf fécondé in vitro, ce qui pourrait être envisagé est le traitement in utero du fœtus par l'injection intra-amniotique de particules virales contenant un gène exogène destiné à remplacer ou à complémenter celui du fotus (voir plus loin). L'avantage d'une telle approche serait de pouvoir être utilisée après un diagnostic prénatal... son désavantage étant de ne s'appliquer qu'à des maladies dont les effets ne seraient pas irréversiblement délétères pour l'embryon et le fotus jeune.

Plus proches sont probablement les premières tentatives de correction post-natale de maladies génétiques monogéniques par autogreffe de cellules génétiquement recombinées. Les affections types qui pourraient bénéficier d'un tel traitement sont les rares déficits enzymatiques associés à un déficit immunitaire sévère, tel le déficit en adénosine désaminase. Une restitution partielle de l'activité enzymatique serait suffisante pour restaurer l'immunité. Il devrait donc être possible de prélever des cellules souches médullaires des malades, de leur transférer un gène (ou un $\mathrm{ADN}$ complémentaire, comme dans le schéma de la figure $1 B$, page suivante) codant pour l'adénosine désaminase, et 


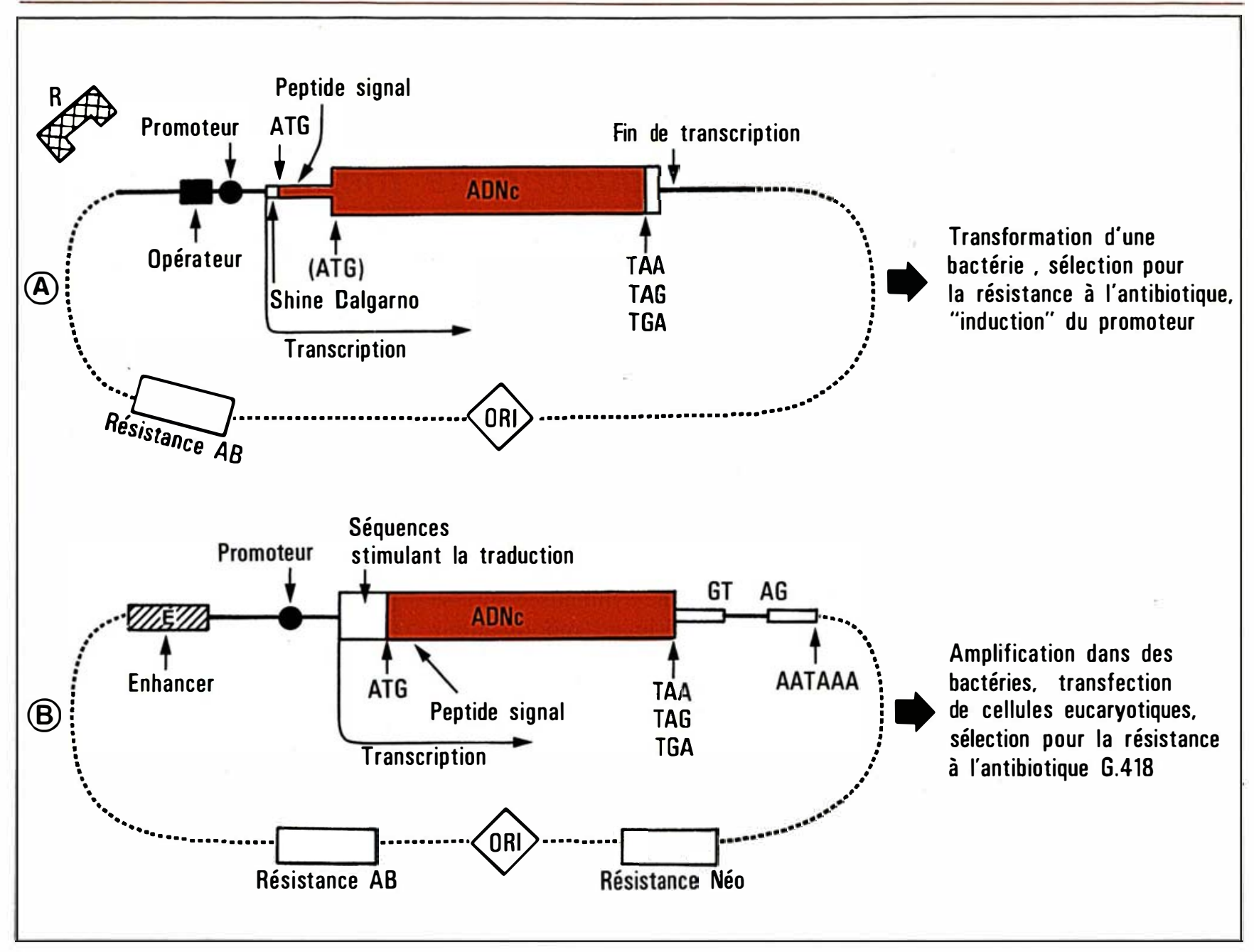

de regreffer aux malades ces cellules ainsi modifiées.

Selon le même principe de restitution partielle, on pourrait envisager de transférer dans des cellules variées (fibroblastes ou cellules médullaires) un ADN complémentaire codant pour un facteur antihémophilique, la sécrétion dans le sang circulant d'une faible quantité d'un tel facteur pouvant mettre les hémophiles à l'abri des accidents hémorragiques les plus graves. Une telle manipulation dans le cas des maladies moléculaires de l'hémoglobine, autrement plus fréquentes que les déficits immunitaires secondaires à une enzymopathie, est beaucoup

modifier ici toutes les cellules souches de telle sorte qu'elles produisent une quantité normale d'hémoglobine normale... ce qui semble aujourd'hui difficile.

Les vecteurs les plus étudiés actuellement pour introduire un gène exogène dans des cellules, médullaires ou autres, sont des rétrovirus dont tous les gènes potentiellement pathogènes ont été délétés et remplacés par le gène à insérer dans le génome des cellules à modifier.

Enfin, une dernière voie théorique de transfert de gène dans l'organisme serait d'injecter au receveur des particules virales infectieuses contenant un génome recombiné et capables de l'introduire dans différentes cellules. Ces particules pourraient être, là encore, rétrovirales, ou bien dérivées de virus à $\mathrm{ADN}$ provoquant normalement des infections bénignes ou inapparentes (certains adénovirus, ou des virus associés aux adénovirus). Les obstacles sont nombreux et divers, comportant le risque qu'un rétrovirus, même non pathogène par lui-même, s'insère à proximité d'un oncogène et l'active, et les incertitudes persistent sur l'avenir d'une infection par un virus à ADN dont le cycle normal ne comporte pas d'intégration dans le génome cellulaire et contre lequel l'organisme doit développer une réaction immunitaire.

A. $\mathbf{K}$. m/s $n^{\circ} 10$ vol. 2, décembre 86 
Figure 1. Construction de gènes artificiels à visée biotechnologique. A) Transformation de cellules bactériennes. L'ADN complémentaire du messager que l'on veut exprimer à un haut niveau dans les cellules bactériennes len général E. coli I est placé sous le contrôle d'un promoteur bactérien inductible et fort. II comprend, au-delà du site d'initiation de la transcription : la séquence de Shine Dalgarno, indispensable à la fixation des ribosomes bactériens au messager, et donc à sa traduction ; un codon d'initiation de la traduction (ATG); une séquence codant pour un peptide signal d'une protéine bactérienne, permettant la sécrétion du produit final du gène étudié dans le milieu de culture bactérienne; éventuellement, un ATG codant pour une méthionine et non utilisé comme initiateur de la traduction, situé juste entre les séquences bactériennes à gauche (rectangle étroit) et eucaryotiques à droite (rectangle large). Au-delà du codon de terminaison de la traduction (TAA, TAG ou TGA), un signal spécifique de fin de transcription est indispensable dans un système procaryotique. Le plasmide dans lequel est intégré ce gène comprend au moins une origine de replication (ORI) et un gène de résistance aux antibiotiques. Les bactéries sont "transformées " par un tel plasmide recombinant qui leur confère la résistance à un antibiotique, ceci permettant d'éliminer les bactéries non transformées. Le gène hybride n'est alors pas exprimé, le promoteur étant inhibé par la fixation d'un répresseur (R) sur une région régulatrice appelée "opérateur". Lorsque cette répression cesse (par exemple, parce que le répresseur est thermo-instable et que le milieu de culture est placé à une température le détruisant, ou bien par addition d'une substance bloquant la fixation du répresseur sur l'opérateur), le gène est transcrit très activement et l'ARN est traduit. Le produit protéique, grâce à la présence d'un peptide signal de protéine bactérienne $\left(\mathrm{m} / \mathrm{s} \mathrm{n}^{\circ} 6\right.$, vol. 2 , $\mathrm{p}$, 341) est sécrété dans le milieu à partir duquel il peut être purifié. Les restes de protéine bactérienne persistant après l'excision du peptide signal lors du passage transmembranaire du produit peuvent être éliminés par clivage de la méthionine rajoutée entre les séquences procaryotiques et eucaryotiques... à condition qu'il n'existe pas d'autres méthionines dans le gène.

B) Transfection de cellules eucaryotiques. Le gène lou plus souvent l'ADN complémentaire dépourvu d'introns) dont on désire faire synthétiser le produit par des cellules eucaryotiques est placé sous le contrôle d'un promoteur et d'une séquence stimulatrice (enhancer) forts et actifs chez les eucaryotes, tels par exemple des promoteurs et des enhancers viraux (LTR du virus de Rous, séquences régulatrices de gènes d'adénovirus ou de virus SV-40). Au-delà du site $d^{\prime}$ initiation de la transcription, on peut introduire dans cette construction plusieurs types de signaux: une séquence localisée dans la partie $5^{\prime}$ non codante du messager et destinée à en augmenter la traductibilité, comme cela a été démontré dans le virus LAV-HTLVIII lou HIV) (séquence TAR, $\mathrm{m} / \mathrm{s} \mathrm{n}^{\circ} 5$, vol. 2, p. 285). Au-delà de l'ATG initiateur de la traduction il est, là aussi, possible d'introduire une séquence de peptide signal permettant la sécrétion du produit dans le milieu; après le signal de fin de traduction, l'introduction d'une séquence comportant un intron, avec ses séquences consensus d'excision-épissage $\left(\mathrm{m} / \mathrm{s} \mathrm{n}^{\circ} 3\right.$, vol. $\left.1, \mathrm{p} .158\right)$ est de nature à augmenter l'expression du gène hybride; enfin, la présence d'un signal de polyadénylation AATAAA est absolument indispensable à la stabilité du messager. Le plasmide dans lequel est insérée cette construction peut être similaire au précédent (ce qui permet son " amplification " dans des cellules bactériennes) et comporter en plus le gène "Néo " qui code pour la résistance des cellules eucaryotiques à l'antibiotique G 418. Ainsi, après amplification du plasmide dans des cellules bactériennes, celui-ci est transféré par divers procédés (on parle alors de "transfection ") dans des cellules eucaryotiques en culture. Le plasmide s'intègre alors au bout de quelques jours dans I'ADN chromosomique des cellules transfectées qui sont sélectionnées grâce à leur résistance au G 418. Les "clones" ainsi isolés sont testés pour la production de la protéine codée par le gène introduit.

$\square \square \square$ BRÈVES $\square \square \square$

$\square$ Cachectine, "tumor necrosis factor" et un facteur induisant la différenciation des myéloblastes en macrophages sont une seule et même molécule sécrétée par les lymphocytes $\mathrm{T}$ stimulés par la phytohémagglutinine. Il s'agit donc d'une lymphokine qui pourrait lyser certaines cellules tumorales (activité "TNF"), changer l'activité de nombreuses enzymes lipogéniques dans les adipocytes (activité " cachectine ") et stimuler la différenciation de cellules souches en macrophage (activité "differentiating inducing factor, DIF ").

[Takeda K et al. Nature 1986 ; 323: 338-40.]

$\mathrm{m} / \mathrm{s} n^{\circ} 10$ vol. 2, décembre 86 $\square \square$ Le gène codant pour l'oncogène c-myc a 3 exons, les 2 derniers codant pour la protéine myc. La séquence du $1^{\text {er }}$ exon révèle que, chez l'homme au moins, il pourrait coder pour une protéine à la fonction encore inconnue. L'équipe de Galibert vient de démontrer qu'une telle protéine existe dans les tissus humains : elle est reconnue par des anticorps dirigés contre des peptides synthétisés d'après la séquence codante supposée de cet exon. L'intervention de cette protéine dans la régulation de l'expression ou de la fonction de l'oncogène c-myc reste hypothétique.

[Gazin C et al. Embo J $1986 ; 5$ : 2241-50.] $\square$ La stabilité des ARN messagers peut être considérablement diminuée in vivo par l'adjonction d'une séquence riche en acide adénylique et uridylique (poly A-U) au niveau de leurs régions 3' non codantes. Cette instabilité exige une synthèse protéique active, suggérant que la séquence poly A-U pourrait être reconnue par une protéine indispensable à la dégradation de l'ARN. Il pourrait s'agir là d'une cible des processus de régulation de l'expression des gènes par modification de la stabilité des transcrits.

[Shaw G, Kamen R, Cell 1986 ; 46 : 659-67.] 